\title{
Solitary Waves and Property Management of Nonlinear Dispersive and Flattened Optical Fiber
}

\author{
Christian Ngouo Tchinda ${ }^{1,2}$, Jean Roger Bogning, 4, * \\ ${ }^{1}$ Department of Physics, Faculty of Science, University of Yaoundé I, Yaoundé, Cameroon \\ ${ }^{2}$ African Center of Excellence in Information Technology and Telecommunications, The University of Yaoundé I, Yaoundé, Cameroon \\ ${ }^{3}$ Department of Physics, Higher Teacher Training College, University of Bamenda, Bamenda, Cameroon \\ ${ }^{4}$ African Optical Fiber Family, Camtel Kamkop Bafoussam, Bafoussam, Cameroon
}

Email address:

rbogning@yahoo.com (J. R. Bogning), ngouochristian@gmail.com (C. N. Tchinda)

${ }^{*}$ Corresponding author

\section{To cite this article:}

Christian Ngouo Tchinda, Jean Roger Bogning. Solitary Waves and Property Management of Nonlinear Dispersive and Flattened Optical Fiber. American Journal of Optics and Photonics. Vol. 8, No. 1, 2020, pp. 27-32. doi: 10.11648/j.ajop.20200801.13

Received: November 21, 2019; Accepted: December 11, 2019; Published: April 13, 2020

\begin{abstract}
In this work, we establish the Conditions that must satisfy the characteristic coefficients of the nonlinear and flattened dispersive optical fiber so that certain classes of solitary waves propagate there with fewer fluctuations. Once the conditions are established, we determine the exact solutions as well as the corresponding nonlinear partial differential equations that govern the propagation dynamics in this transmission medium. The propagation of the solutions obtained is also tested. The method used to obtain the analytical solutions is based on the control of the properties of the Bogning implicit functions whereas the numerical simulations are made through the split-step method which is very adapted to simulate the propagation of the signals.
\end{abstract}

Keywords: Flattened Optical Fiber, Solitary Wave, Characteristic Coefficient, Implicit Bogning Function, Propagation, Nonlinear, Dispersive, Partial Differential Equation

\section{Introduction}

Nonlinear physics in its branch of photonic optics has been at the center of many telecommunications technology applications in recent years. Among the transmission media and waveguides developed by these technologies, optical fiber is attracting even more interest, probably because of its high bandwidth and insensitivity to external electromagnetic disturbances. This great interest is also reflected by the large number of research works devoted to this ultimate transmission medium. The propagation dynamics of the waves in the fiber is generally modeled by partial differential equations of Schrödinger type with nonlinear terms, dispersion terms and dissipation terms characterized by their coefficients [1-5]. If the coefficient of nonlinearity is responsible for the unpredictable effects that the propagating wave may undergo, the dispersion coefficient is responsible for the spread of the signal and the dissipation coefficient responsible for the absorption or losses of the energy. These observations and remarks assume that these effects must be taken into account during the fabrication of an optical fiber capable of better transmitting a signal. There are several types of optical fibers but the optical fiber that will be the focus of our study in this paper is the nonlinear dispersive and flattened optical fiber. The main characteristic of this one is that it is subject to strong dispersions and this can be understood because of its flattened shape. The nonlinear partial differential equation that models the propagation dynamics of waves in this type of fiber generally has higher order dispersion terms (greater than or equal to 4). Thus, the work that we have defined consists in establishing the constraint relations which make it possible to determine the type of wave (solitary wave) likely to propagate without difficulties in the waveguide. once these conditions are fixed, we make corrections to the initial differential equation to obtain an equation which admits for exact solution the analytical sequence attributed to the wave that must propagate in the optical fiber [6-8]. To achieve this, we will first assume that the fiber is immersed in a medium with 
arbitrary characteristic coefficients $n_{i}(i=1,2,3,4)$ and subsequently find a uniting relation such that the desired solution is effective. The method and technique used to obtain the results is the one we develop in recent years in our many works [9-33].

This article is organized as follows: Section 2 establishes coefficient range equations as well as the range of possibilities for obtaining solutions. In Section 3, we propose analytical solutions as well as constraint relations for this to be possible. In section 4, we numerically verify the propagation of solitary wave solutions obtained and finally we end the work with a conclusion.

\section{Equation of Range of Coefficients and Possibilities of Solutions}

We define the family of nonlinear partial differential equations that describes the propagation dynamics of the wave in a dispersive and flattened optical fiber as

$$
i n_{1} \frac{\partial U}{\partial \xi}+n_{2} \frac{\partial^{2} U}{\partial \tau^{2}}+n_{3}|U|^{2} U+n_{4} \frac{\partial^{4} U}{\partial \tau^{4}}=0
$$

where $n_{i}(i=1,2,3,4)$ are the characteristic coefficients of the fiber, $\xi$ is the spatial variable and $\tau$ is the temporal variable. The flattening of the fiber is materialized hereby the partial derivative of order $4\left(\frac{\partial^{4} U}{\partial \tau^{4}}\right)$. We propose to construct the solution of equation (1) in the form

$$
U(\xi, \tau)=A(\tau) \exp i k \xi
$$

where $A(\tau)$ is the temporal envelope of the wave solution and $k$ the wave number. The introduction of the ansatz (2) in equation (1) gives

$$
-n_{1} k A+n_{2} \frac{\partial^{2} A}{\partial \tau^{2}}+n_{3}|A|^{2} A+n_{4} \frac{\partial^{4} A}{\partial \tau^{4}}=0
$$
form

We propose to construct the solution of equation (3) in the

$$
A(\tau)=a J_{n, m}(\alpha \tau)
$$

where $a$ is a constant to be determined, $J_{n, m}(\alpha \tau)$ the implicit function whose numbers $n$ and $m$ are real to be determined and $\alpha$ a parameter to be determined as well. Thus, taking into account equation (4) in (3) gives

$$
\begin{aligned}
& -n_{1} k a J_{n, m}+n_{2} \alpha^{2} a\left[m(m-1) J_{n-2, m-2}-(2 m n-m+n) J_{n, m}+n(n+1) J_{n+2, m+2}\right] \\
& +n_{3}|a|^{2} a J_{3 n, 3 m}+n_{4} \alpha^{4} a m(m-1)(m-2)(m-3) J_{n-4, m-4}-n_{4} \alpha^{4} a m(m-1)(m-2)(n-3) J_{n-2, m-2} \\
& +n_{4} \alpha^{4} a[m(m-1)(n-2)+(2 m n-m+n) m](m-1) J_{n-2, m-2} \\
& +n_{4} \alpha^{4} a[m(m-1)(n-2)+(2 m n-m+n) m](n-1) J_{n, m}-n_{4} \alpha^{4} a[(2 m n-m+n) n+n(n+1)(m+2)](m+1) J_{n, m} \\
& +n_{4} \alpha^{4} a[(2 m n-m+n) n+n(n+1)(m+2)](m+1) J_{n, m}+n_{4} \alpha^{4} a[(2 m n-m+n) n+n(n+1)(m+2)](n+1) J_{n+2, m+2} \\
& -n_{4} \alpha^{4} a n(n+1)(n+2)(m+3) J_{n+2, m+2}+n_{4} \alpha^{4} a n(n+1)(n+2)(n+3) J_{n+4, m+4}=0
\end{aligned}
$$

This equation is generally called the range equation of the coefficients, since it represents the equation whose analysis makes it possible to determine the coefficients. In the case of equation (5), the only coefficient to be determined is $a$. The different equations to be solved depend on the values assigned to $n$ and $m$.

But the choice of the values of $n$ and $m$ is not hazardous; we have established in our previous work that the values of $n$ and $m$ for which are listed among the values of $n$ and $m$ for which some terms of equation (5) are grouped together. Thus, the values of $n$ and $m$ for which some terms of equation (5) are related are given by

$$
n, m \in\left\{-2,-\frac{3}{2},-1,-\frac{1}{2}, 0, \frac{1}{2}, 1, \frac{3}{2}, 2\right\}
$$

The various combinations of the pair $(n, m)$ likely to lead to solutions are recorded in the table below called fields of possibilities for finding solutions.

Table 1. Fields of possibilities for finding solutions.

\begin{tabular}{llllllllll}
\hline$(\boldsymbol{n}, \boldsymbol{m})$ & $-\mathbf{2}$ & $-\frac{\mathbf{3}}{\mathbf{2}}$ & $-\mathbf{1}$ & $-\frac{\mathbf{1}}{\mathbf{2}}$ & $\mathbf{0}$ & $\mathbf{1}$ & $\mathbf{1}$ & $\mathbf{3}$ & $\mathbf{2}$ \\
\hline-2 & $(-2,-2)$ & $\left(-2,-\frac{3}{2}\right)$ & $(-2,-1)$ & $\left(-2,-\frac{1}{2}\right)$ & $(-2,0)$ & $\left(-2, \frac{1}{2}\right)$ & $(-2,1)$ & $\left(-2, \frac{3}{2}\right)$ & $(-2,2)$ \\
$-\frac{3}{2}$ & $\left(-\frac{3}{2},-2\right)$ & $\left(-\frac{3}{2},-\frac{3}{2}\right)$ & $\left(-\frac{3}{2},-1\right)$ & $\left(-\frac{3}{2},-\frac{1}{2}\right)$ & $\left(-\frac{3}{2}, 0\right)$ & $\left(-\frac{3}{2}, \frac{1}{2}\right)$ & $\left(-\frac{3}{2}, 1\right)$ & $\left(-\frac{3}{2}, \frac{3}{2}\right)$ & $\left(-\frac{3}{2}, 2\right)$ \\
-1 & $(-1,-2)$ & $\left(-1,-\frac{3}{2}\right)$ & $(-1,-1)$ & $\left(-1,-\frac{1}{2}\right)$ & $(-1,0)$ & $\left(-1, \frac{1}{2}\right)$ & $(-1,1)$ & $\left(-1, \frac{3}{2}\right)$ & $(-1,2)$ \\
\hline
\end{tabular}




\begin{tabular}{|c|c|c|c|c|c|c|c|c|c|}
\hline$(n, m)$ & -2 & $-\frac{3}{2}$ & -1 & $-\frac{1}{2}$ & 0 & $\frac{1}{2}$ & 1 & $\frac{3}{2}$ & 2 \\
\hline$-\frac{1}{2}$ & $\left(-\frac{1}{2},-2\right)$ & $\left(-\frac{1}{2},-\frac{3}{2}\right)$ & $\left(-\frac{1}{2},-1\right)$ & $\left(-\frac{1}{2},-\frac{1}{2}\right)$ & $\left(-\frac{1}{2}, 0\right)$ & $\left(-\frac{1}{2}, \frac{1}{2}\right)$ & $\left(-\frac{1}{2}, 1\right)$ & $\left(-\frac{1}{2}, \frac{3}{2}\right)$ & $\left(-\frac{1}{2}, 2\right)$ \\
\hline 0 & $(0,-2)$ & $\left(0,-\frac{3}{2}\right)$ & $(0,-1)$ & $\left(0,-\frac{1}{2}\right)$ & $(0,0)$ & $\left(0, \frac{1}{2}\right)$ & $(0,1)$ & $\left(0, \frac{3}{2}\right)$ & $(0,2)$ \\
\hline$\frac{1}{2}$ & $\left(\frac{1}{2},-2\right)$ & $\left(\frac{1}{2},-\frac{3}{2}\right)$ & $\left(\frac{1}{2},-1\right)$ & $\left(\frac{1}{2},-\frac{1}{2}\right)$ & $\left(\frac{1}{2}, 0\right)$ & $\left(\frac{1}{2}, \frac{1}{2}\right)$ & $\left(\frac{1}{2}, 1\right)$ & $\left(\frac{1}{2}, \frac{3}{2}\right)$ & $\left(\frac{1}{2}, 2\right)$ \\
\hline 1 & $(1,-2)$ & $\left(1,-\frac{3}{2}\right)$ & $(1,-1)$ & $\left(1,-\frac{1}{2}\right)$ & $(1,0)$ & $\left(1, \frac{1}{2}\right)$ & $(1,1)$ & $\left(1, \frac{3}{2}\right)$ & $(1,2)$ \\
\hline$\frac{3}{2}$ & $\left(\frac{3}{2},-2\right)$ & $\left(\frac{3}{2},-\frac{3}{2}\right)$ & $\left(\frac{3}{2},-1\right)$ & $\left(\frac{3}{2},-\frac{1}{2}\right)$ & $\left(\frac{3}{2}, 0\right)$ & $\left(\frac{3}{2}, \frac{1}{2}\right)$ & $\left(\frac{3}{2}, 1\right)$ & $\left(\frac{3}{2}, \frac{3}{2}\right)$ & $\left(\frac{3}{2}, 2\right)$ \\
\hline 2 & $(2,-2)$ & $\left(2,-\frac{3}{2}\right)$ & $(2,-1)$ & $\left(2,-\frac{1}{2}\right)$ & $(2,0)$ & $\left(2, \frac{1}{2}\right)$ & $(2,1)$ & $\left(2, \frac{3}{2}\right)$ & $(2,2)$ \\
\hline
\end{tabular}

\section{Analytical Solutions and Constraint Relationships}

In this section, we look for solutions corresponding to the different values of the pair $(n, m)$ of the table 1 .

1. case $(n, m)=(2,0)$ : We obtain from equation (5), the following equation

$$
-2 n_{2} \alpha^{2} a J_{2,0}+6 n_{2} \alpha^{2} a J_{4,2}-n_{1} k a J_{2,0}+n_{3}|a|^{2} a J_{6,0}-42 n_{4} \alpha^{4} a J_{4,2}+120 n_{4} \alpha^{4} a J_{6,4}=0
$$

Knowing that $J_{4,2}=J_{2,0}-J_{4,0}$ and $J_{6,4}=J_{2,0}-2 J_{4,0}+J_{6,0}$, equation (7) becomes

$$
\left(4 n_{2} \alpha^{2} a-n_{1} k a+78 n_{4} \alpha^{4} a\right) J_{2,0}+\left(-6 n_{2} \alpha^{2} a-198 n_{4} \alpha^{4} a\right) J_{4,0}+\left(n_{3}|a|^{2} a+120 n_{4} \alpha^{4} a\right) J_{6,0}=0
$$

Equation (8) is verified if for $a \neq 0$, we have

$$
\begin{gathered}
n_{2}=-33 n_{4} \alpha^{2} \\
|a|=\alpha \sqrt{\frac{-120 n_{4}}{n_{3}}}, n_{4} n_{3} \prec 0, \alpha \succ 0
\end{gathered}
$$$$
4 n_{2} \alpha^{2}-n_{1} k+78 n_{4} \alpha^{4}=0
$$$$
n_{2}+33 n_{4} \alpha^{2}=0
$$$$
n_{3}|a|^{2}+120 n_{4} \alpha^{2}=0
$$

The resolution of equations (9), (10) and (11) allows having

$$
n_{1}=-\frac{54 n_{4} \alpha^{4}}{k}
$$

We deduce from equation (14) that

$$
a=\alpha \sqrt{\frac{-120 n_{4}}{n_{3}}} \exp i \theta, \theta \in R
$$

The sought solution is given by

$$
U(\xi, \tau)=\alpha \sqrt{\frac{-120 n_{4}}{n_{3}}} J_{2,0}(\alpha \tau) \exp i(k \xi+\theta), n_{4} n_{3} \prec 0, \theta \in R
$$

Solution (15) is the exact solution of the equation below obtained by modifying the coefficients of equation (1)

$$
54 i n_{1} \alpha^{4} \frac{\partial U}{\partial \xi}+33 k n_{4} \alpha^{2} \frac{\partial^{2} U}{\partial \tau^{2}}-k n_{3}|U|^{2} U-k n_{4} \frac{\partial^{4} U}{\partial \tau^{4}}=0
$$

2. case $(n, m)=(2,1)$ : We obtain from equation (5), the following equation

$$
\left(5 n_{2} \alpha^{2} a+n_{1} k a+23 \alpha^{4} n_{4} a\right) J_{2,1}-\left(6 n_{2} \alpha^{2} a-12 n_{4} \alpha^{4} a\right) J_{4,3}-n_{3}|a|^{2} a J_{6,3}-96 n_{4} \alpha^{4} a J_{6,5}=0
$$


transformations

$$
\begin{gathered}
J_{4,3}=J_{2,1}-J_{4,1} \\
J_{6,3}=J_{4,1}-J_{6,1} \\
\left.\alpha^{2} a-61 n_{4} \alpha^{4} a+n_{1} k a\right) J_{2,1} \\
\text { verified if we have } \\
|a|=4 \alpha^{2} \sqrt{\frac{6 n_{4}}{n_{3}}}, n_{4} n_{3} \succ 0 \\
n_{2}=-14 n_{4} \alpha^{2} \\
k=\frac{47 n_{4} \alpha^{2}}{n_{1}}, n_{1} \neq 0
\end{gathered}
$$$$
\left(-n_{2} \alpha^{2} a-61 n_{4} \alpha^{4} a+n_{1} k a\right) J_{2,1}+\left(6 n_{2} \alpha^{2} a+180 n_{4} \alpha^{4} a-n_{3}|a|^{2} a\right) J_{4,1}+\left(n_{3}|a|^{2} a-96 n_{4} \alpha^{4} a\right) J_{6,1}=0
$$

Equation (21) is verified if we have

We obtain from equation (23)

$$
a=4 \alpha^{2} \sqrt{\frac{6 n_{4}}{n_{3}}} \exp i \theta, \theta \in R
$$

Taking into account the relationships and constraints above allows to write the solution

$$
U(\xi, \tau)=4 \alpha^{2} \sqrt{\frac{6 n_{4}}{n_{3}}} J_{2,1}(\alpha \tau) \exp i\left(\frac{47 n_{4} \alpha^{4}}{n_{1}} \xi+\theta\right)
$$

Equation (26) is the exact solution of the corrected equation

$$
i n_{1} \frac{\partial U}{\partial \xi}-14 n_{4} \alpha^{2} \frac{\partial^{2} U}{\partial \tau^{2}}+n_{3}|U|^{2} U+n_{4} \frac{\partial^{4} U}{\partial \tau^{4}}=0
$$

3. $\operatorname{case}(n, m)=(-2,0)$ : We obtain from equation (5), the following equation

$$
\left(2 n_{2} \alpha^{2}-n_{1} k\right) J_{-2,0}+\left(2 n_{2} \alpha^{2}-4 n_{4} \alpha^{4}\right) J_{0,2}+n_{3}|a|^{2} a J_{-6,0}=0
$$

Equation (29) is verified if and only if $n_{2}=2 n_{4} \alpha^{2}, n_{3}=0$ and $k=\frac{4 n_{4} \alpha^{4}}{n_{1}}$ with $n_{1} \neq 0$. In these conditions, the seeking solution is given by

$$
U(\xi, \tau)=a J_{-2,0}(\alpha \tau) \exp \frac{4 i n_{4} \alpha^{4}}{n_{1}} \xi, n_{1} \neq 0
$$

Equation (30) is the exact solution of the following partial differential equation

$$
i n_{1} \frac{\partial U}{\partial \xi}+2 n_{4} \alpha^{2} \frac{\partial^{2} U}{\partial \tau^{2}}+n_{4} \frac{\partial^{4} U}{\partial \tau^{4}}=0
$$

$$
J_{6,5}=J_{2,1}-2 J_{4,1}+J_{6,1}
$$

Taking into account relations (18), (19) and (20) in equation (17) leads to

We can see that equation (30) describes the propagation dynamics in the very weakly nonlinear flattened optical fiber $\left(n_{3} \rightarrow 0\right)$.

\section{Numerical Study}

In this section, we use the split-step method [34] to discretize the nonlinear partial differential equations (17) and (28) and to propagate their corresponding solutions. Thus, the constraint relations between the coefficients of the terms of the nonlinear partial differential equation allowed choosing the values of the parameters. We organized this numerical study in two cases.

1. First case

The nonlinear partial differential equation (17) is discretized so that the envelope $U(\xi, \tau)$ is given by the relation (16). The profiles obtained are as follows
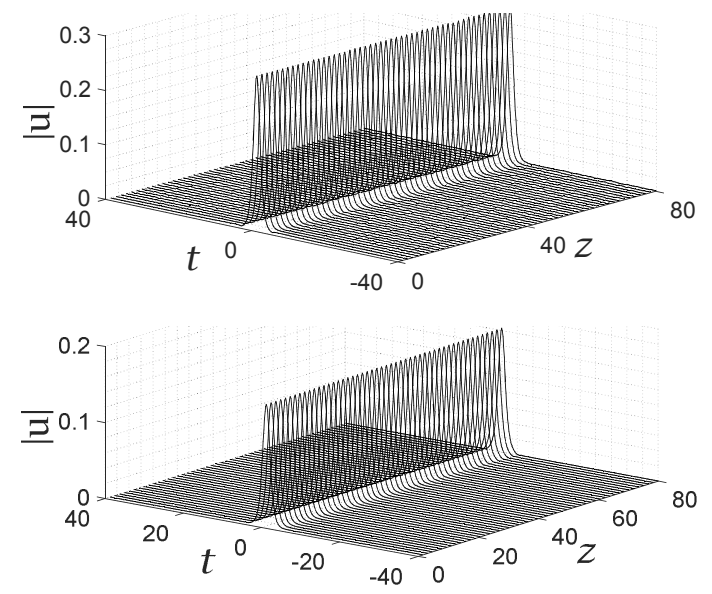

Figure 1. Propagation of the solitary wave (16) in equation (17): the left profile is obtained for: $n_{4}=0.01, n_{1}=0.01, n_{3}=-10, \alpha=0.8, \theta=\pi$ the right profile is obtained for $n_{4}=0.5, n_{1}=20, n_{3}=-2, \alpha=0.2, \theta=\pi / 6$.

\section{Second case}

The nonlinear partial differential equation (17) is discretized so that the envelope $U(\xi, \tau)$ is given by the relation (16). The profiles obtained are as follows 

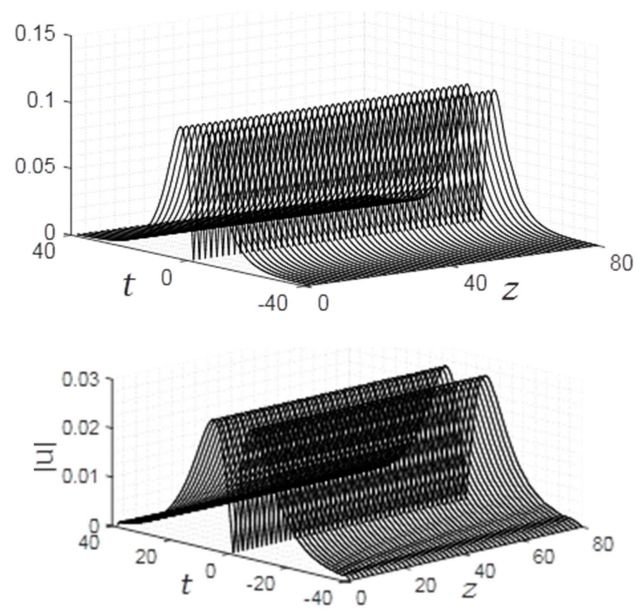

Figure 2. Propagation of the solitary wave (16) in equation (17): the left profile is obtained for: $n_{4}=0.5, n_{1}=20, n_{3}=-2, \alpha=0.2, \theta=\pi / 6$ the right profile is obtained for $n_{4}=0.1, n_{1}=5, n_{3}=-1, \alpha=0.13, \theta=\pi$.

\section{Conclusion}

We have in the framework of this article studied how to choose the characteristic parameters of the single-mode fiber flattened so that the differential equations that govern the propagation dynamics in this transmission medium admit desired solutions. To achieve this, we divide the work into two major parts. A first part, where we have analytically established the relationships linking the parameters of the fiber or medium in which the fiber is immersed, so that the solutions we need have been obtained. To this end, we assigned the coefficients $n_{i}(i=1,2,3,4)$ to the different terms of the nonlinear partial differential equation to solve and subsequently obtain the constraints that bind the $n_{i}$ coefficientsto $n_{i}$ and other parameters of the studied system. We have found a field of possibilities of obtaining solutions through the different values that can take $n$ and $m$. We note that in the case of the flattened optical fiber that is to say highly dispersive, the solitary wave solutions obtained are pulse of second order and kink of second order. All the values of the pairs $(n, m)$ do not lead to the important solutions in physics domain. We get a second-order pulse for the pair $(2,0)$ and a second-order kink solution for the pair $(2,1)$. The split-step method is used to study the propagation of the solutions obtained. The study in this paper is very fascinating and interesting analytically because beyond its physical reach, it has aconsiderable mathematical significance. Numerically, we have checked the reliability of the solutions obtained. This study can potentially have very positive impacts in propagation phenomena in the optical fiber and naturally in mathematics for nonlinear physics.

\section{References}

[1] A. Hasegawa and F. Tappert, (1973), Transmission of stationary nonlinear optical pulses in dielectric fibers in anomalous dispersion Applied Physisc Letters, 23, 142-144.
[2] L. F. Mollenauer, R. F. Stolen and J. P. Gordon, (1980), Experimental observation of picoseconds pulse narrowing and solitons in optical fibers Physics Review Letters, 45, 1095.

[3] R. Hirota, (1971), Exact solution of the KdV equation for multiple collisions of solitons Physics Review Letters, 27, 1192-1194.

[4] G. P. Agrawal, (2012) Nonlinear Fibre Optics, Academic Press.

[5] G. P. Agrawal, (2012), Applications of Nonlinear Fibre Optics, Academic Press.

[6] J. R. Bogning, (2019), Mathematics for nonlinear Physics: solitary waves in the center of resolution of dispersive nonlinear partial differential equations, Dorrance Publishing Co, USA.

[7] J. R. Bogning, (2019), Mathematics for Physics: The implicit Bogning functions and applications, Lambert Academic Publishing, Germany.

[8] Jean roger Bogning, (2018), Exact solitary wave solutions of $(3+1)$ modified B-type Kadomtsev-Petviashvili family equations, American Journal of Computational and Applied Mathematics, 8 (5), 85-92.

[9] J. R. Bogning, C. T. Djeumen Tchaho and T. C. Kofané, (2012), Construction of the soliton solutions of the GinzburgLandau equations by the new Bogning-Djeumen TchahoKofané method, Physica Scripta, 85, 025013-025018.

[10] J. R. Bogning, C. T. Djeumen Tchaho and T. C. Kofané, (2012), Generalization of the Bogning- Djeumen TchahoKofane Method for the construction of the solitary waves and the survey of the instabilities, Far East Journal of Dynamical Systems, 20 (2), 101-119.

[11] J. R. Bogning, C. T. Djeumen Tchaho and T. C. Kofané, (2013), Solitary wave solutions of the modified Sasa- Satsuma nonlinear partial differential equation American Journal of Computational and Applied Mathematics, 3 (2), 97-107.

[12] J. R. Bogning, (2013), Pulse soliton solutions of the modified $\mathrm{KdV}$ and Born-Infeld equations, International Journal of Modern Nonlinear Theory and Application, 2, 135-140.

[13] J. R. Bogning, K. Porsezian, G. Fautso Kuiaté, H. M. Omanda, (2015), gap solitary pulses induced by the Modulational instability and discrete effects in array of inhomogeneous optical fibers, Physics Journal, 1 (3), 216-224.

[14] J. R. Bogning, (2015), $\mathrm{N}^{\text {th }}$ Order Pulse Solitary Wave Solution and Modulational Instability in the Boussinesq, Equation American Journal of Computational and Applied Mathematics, 5 (6), 182-188.

[15] J. R. Bogning, (2015) $\mathrm{Sech}^{\mathrm{n}}$ Solutions of the generalized and modified Rosenau-Hyman Equations, Asian Journal of Mathematics and Computer Research, 9 (1), 2395-4205.

[16] J. R. Bogning, C. T. Djeumen Tchaho and H. M. Omanda, (2016), Combined solitary wave solutions in higher-order effects optical fibers, British Journal of Mathematics and Computer Science, 13 (3), 1-12.

[17] C. T. Djeumen Tchaho, J. R. Bogning and T. C. Kofané, (2012), Modulated Soliton Solution of the Modified Kuramoto-Sivashinsky's Equation, American Journal of Computational and Applied Mathematics, 2 (5), 218-224. 
[18] C. T. Djeumen Tchaho, J. R. Bogning and T. C. Kofane, (2011), Multi-Soliton solutions of the modified KuramotoSivashinsky's equation by the BDK method, Far East Journal of Dynamical systems, 15 (2), 83-98.

[19] C. T. Djeumen Tchaho, J. R. Bogning and T. C. Kofane, (2010), Construction of the analytical solitary wave solutions of modified Kuramoto-Sivashinsky equation by the method of identification of coefficients of the hyperbolic functions, Far East Journal of dynamical systems, 14 (1), 14-17.

[20] J. R. Bogning, G. Fautso Kuiaté, H. M. Omanda and C. T. Djeumen Tchaho, (2015), Combined Peakons and multiplepeak solutions of the Camassa-Holm and modified KdV equations and their conditions of obtention, Physics Journal, 1 (3), 367-374

[21] J. R. Bogning, (2013), Analytical soliton solutions and wave solutions of discrete nonlinear cubic-quintique GinzburgLandau equations in array of dissipative optical system,. American Journal of Computational and Applied Mathematics, 3 (2), 97-105.

[22] R. Njikue, J. R. Bogning and T. C. Kofane, (2018), Exact bright and dark solitary wave solutions of the generalized higher order nonlinear Schrödinger equation describing the propagation of ultra-short pulse in optical fiber, J. Phys. Commun, 2, 025030.

[23] J. R. Bogning and T. C. Kofané, (2006), Solitons and dynamics of nonlinear excitations in the array of optical fibers, Chaos, Solitons \& Fractals, 27 (2), 377-385.

[24] J. R. Bogning and T. C. Kofané, (2006), Analytical solutions of the discrete nonlinear Schrödinger equation in arrays of optical fibers, Chaos, Solitons \& Fractals, 28 (1), 148-153.

[25] J. R. Bogning, (2018), Exact solitary wave solutions of the $(3+1)$ modified B-type Kadomtsev-Petviashvili family equations, American Journal of computational and applied mathematics, 8 (5), 85-92.

[26] G. Tiague Takongmo and J. R. Bogning, (2018), Construction of solitary wave solutions of higher-order nonlinear partial differential equations modeled in a nonlinear hybrid electrical line, American Journal of circuits, systems and signal processing, 4 (3), 36-44.

[27] G. Tiague Takongmo and J. R, Bogning, (2018), Construction of solitary wave solutions of higher-order nonlinear partial differential equations modeled in a modified nonlinear Noguchi electrical line, American Journal of circuits, systems and signal processing, 4 (1) 8-14.

[28] G. Tiague Takongmo and J. R. Bogning, (2018), Construction of solitary wave solutions of higher-order nonlinear partial differential equations modeled in a nonlinear capacitive electrical line, American Journal of circuits, systems and signal processing, 4 (2), 15-22.

[29] G. Tiague Takongmo and J. R, Bogning, (2018), Construction of solutions in the shape (pulse, pulse) and (kink, kink) of a set of two equations modeled in a nonlinear inductive electrical line with crosslink capacitor, American Journal of circuits, systems and signal processing, 4 (2), 28-35.

[30] G. Tiague Takongmo and J. R. Bogning, (2018), (kink, kink) and (pulse, pulse) exact solutions of equations modeled in a nonlinear capacitive electrical line with capacitor, American Journal of circuits, systems and signal processing, 4 (3), 4553.

[31] G. Tiague Takongmo and J. R. Bogning, (2018), Solitary wave solutions of modified telegraphist equations modeled in an electrical line, Physics Journal, 4 (3), 29-36.

[32] G. Tiague Takongmo and J. R. Bogning, (2018), Coupled soliton solutions of modeled equations in a Noguchi electrical line with crosslink capacitor, Journal of Physics communications, 2, 105016.

[33] Rodrique Njikue and J. R. Bogning, T. C. Kofané, (2018), higher order nonlinear Schrödinger equation family in optical fiber and solitary wave solutions, American journal of optics, American Journal of optics and photonics, 6 (3), 31-41.

[34] Ryan Napoleon Foster, 2007, Web based interface for numerical simulations of nonlinear evolution equations. 